\title{
Pelvic Lymphadenectomy
}

National Cancer Institute

\section{Source}

National Cancer Institute. Pelvic Lymphadenectomy. NCI Thesaurus. Code C51896.

Surgical removal of one or more pelvic lymph nodes. 